\title{
Genomic and transcriptomic characterization of the human glioblastoma cell line AHOL1
}

\author{
W.A.S. Ferreira ${ }^{1 \oplus}$, C.K.N. Amorim ${ }^{1 \oplus}$, R.R. Burbano ${ }^{2,3,4 \oplus}{ }^{\text {, R.A.R. Villacis }}{ }^{5 \odot}$, F.A. Marchi ${ }^{6 \oplus}$, \\ T.S. Medina ${ }^{6 \oplus}$, M.M.C. de Lima $^{7 \oplus}$, and E.H.C. de Oliveira ${ }^{1,80}$ \\ ${ }^{1}$ Laboratório de Cultura de Tecidos e Citogenética, SAMAM, Instituto Evandro Chagas, Ananindeua, PA, Brasil \\ ${ }^{2}$ Laboratório de Citogenética Humana, Instituto de Ciências Biológicas, Universidade Federal do Pará, Belém, PA, Brasil \\ ${ }^{3}$ Núcleo de Pesquisas em Oncologia, Hospital Universitário João de Barros Barreto, Belém, PA, Brasil \\ ${ }^{4}$ Laboratório de Biologia Molecular, Hospital Ophir Loyola, Belém, PA, Brasil \\ ${ }^{5}$ Departamento de Genética e Morfologia, Instituto de Ciências Biológicas, Universidade de Brasília, Brasília, DF, Brasil \\ ${ }^{6}$ Centro Internacional de Pesquisa, A.C. Camargo Cancer Center, São Paulo, SP, Brasil \\ ${ }^{7}$ Instituto de Ciências Biológicas, Faculdade de Biomedicina, Universidade Federal do Pará, Belém, PA, Brasi \\ ${ }^{8}$ Instituto de Ciências Exatas e Naturais, Faculdade de Ciências Naturais, Universidade Federal do Pará, Belém, PA, Brasil
}

\begin{abstract}
Cancer cell lines are widely used as in vitro models of tumorigenesis, facilitating fundamental discoveries in cancer biology and translational medicine. Currently, there are few options for glioblastoma (GBM) treatment and limited in vitro models with accurate genomic and transcriptomic characterization. Here, a detailed characterization of a new GBM cell line, namely AHOL1, was conducted in order to fully characterize its molecular composition based on its karyotype, copy number alteration (CNA), and transcriptome profiling, followed by the validation of key elements associated with GBM tumorigenesis. Large numbers of CNAs and differentially expressed genes (DEGs) were identified. CNAs were distributed throughout the genome, including gains at Xq11.1-q28, Xp22.33-p11.1, Xq21.1-q21.33, 4p15.1-p14, 8q23.2-q23.3 and losses at Yq11.21-q12, Yp11.31-p11.2, and 15q11.1-q11.2 positions. Nine druggable genes were identified, including HCRTR2, ETV1, PTPRD, PRKX, STS, RPS6KA6, ZFY, USP9Y, and KDM5D. By integrating DEGs and CNAs, we identified 57 overlapping genes enriched in fourteen pathways. Altered expression of several cancer-related candidates found in the DEGs-CNA dataset was confirmed by RT-qPCR. Taken together, this first comprehensive genomic and transcriptomic landscape of AHOL1 provides unique resources for further studies and identifies several druggable targets that may be useful for therapeutics and biologic and molecular investigation of GBM.
\end{abstract}

Key words: Array-comparative genomic hybridization; Gliomas; Transcriptomics; Brain tumors; Cell line; Glioblastoma

\section{Introduction}

Glioblastomas (GBMs) are heterogeneous primary brain tumors that are likely originated from oligodendrocyte precursor cells, neural stem cells (NSCs), and NSCderived astrocytes. They are the most lethal and common malignancy among all brain tumors, with an incidence rate of 3.21 cases per 100,000 individuals, median survival rate of 12-18 months, and higher predominance in males. GBMs are commonly diagnosed in elderly patients (median of 65 years), increasing with age, peaking at 75-84 years, and declining after 85 years (1).

According to the new classification for central nervous system (CNS) tumors proposed by the World Health Organization (WHO) in 2016, GBMs are classified as grade IV and included in diffuse astrocytic and oligodendroglial tumors. Based on the mutational pattern of the isocitrate dehydrogenase (IDH) gene, they are further classified as i) IDH-wildtype GBM ( $90 \%$ of cases), which frequently correspond to clinically defined primary GBM (or de novo GBM), arising predominately from the supratentorial region in patients with median age of $\sim 62$ years at diagnosis and whose mean length of clinical history is 4 months; or ii) IDH-mutant-type GBM (10\% of cases), which are defined as secondary GBM preferentially arising from the frontal region of younger patients (median age at diagnosis of $\sim 44$ years) and whose prognosis is usually better than those with IDH-wildtype (1).

Currently, standard treatment for both GBM entities encompasses surgical resection followed by radiotherapy and chemotherapy (mainly using temozolomide - TMZ). However, these aggressive treatments are not effective in 
controlling the disease, thus indicating a high demand for new efficacious therapies to improve outcomes of patients with GBM (1).

In vitro cultures of GBM cell lines have been widely used as an important model for understanding GMB heterogeneity, drug sensitivity and resistance, evaluation of new therapeutic approaches, and to search for novel biomarkers. The Human Glioblastoma Cell Culture (HGCC) biobank has assembled a panel of 53 cell lines derived from surgical samples of GBM patients. However, there is a limited number of GBM cell lines deposited in HGCC or other biobanks (Broad-Novartis Cancer Cell Line Encyclopedia and American Type Culture Collection), given the heterogeneity of each molecular subtype of GBM (2). Thus, new, well-characterized cell lines that resemble these different molecular subtypes of GBM are still needed to better comprehend the molecular mechanisms involved in GBM tumorigenesis. To this end, the goal of this study was to characterize the chromosomal composition based on copy number alteration (CNA) and transcriptome profile of a newly established glioblastoma cell line, as a strategy to discover potential druggable targets that might prevent GBM development and progression.

\section{Material and Methods}

\section{Study approval by the Research Ethics Committee and collection of non-neoplastic samples}

This study was approved by the Research Ethics Committee of the Instituto Evandro Chagas/IEC/SVS/MS (Process Number 192.336). The use of the sample to establish the AHOL1 cell line was approved by the Research Ethics Committee of the Ophir Loyola Hospital, and a written informed consent was obtained from the patient.

Ten non-neoplastic samples from nervous tissue were obtained from biopsies of patients from Ophir Loyola Hospital, Belém, Brazil. All tissue samples were immediately frozen in liquid nitrogen and stored in DNA/RNA Shield ${ }^{\mathrm{TM}}$ (Zymo Research, USA) at $-80^{\circ} \mathrm{C}$ until the extraction stage.

\section{Materials}

Dulbecco's modified Eagle's medium (DMEM), fetal bovine serum (FBS), trypsin/EDTA, penicillin G, and streptomycin were obtained from Gibco (USA) and used to grow GBM cell lines in culture.

\section{Culture of human glioblastoma cell lines}

The main subject of this study was the AHOL1 (Astrocytoma Ophir Loyola Hospital 1) cell line established by our group at the Human Cytogenetics Laboratory, Federal University of Pará (UFPA), from a secondary GBM obtained from the tumor resection of a 43-year-old multiracial male patient treated at the Neurological Clinic of Ophir Loyola Hospital (Brazil) with a histopathologic diagnosis of GBM (grade IV) that evolved from a grade III astrocytoma (3).
For comparison to common alterations observed in glioblastoma cell lines, we used three well established human glioblastoma cell lines in our experiments: U-343 MGa cell line kindly provided by the Cytogenetics and Mutagenesis Laboratory, University of São Paulo (Ribeirão Preto, Brazil), derived from a primary GBM from a Caucasian adult patient and obtained from CLS Cell Lines Service (CLS order number 300365), U-87 MG purchased from American Type Culture Collection (catalog number ATCC ${ }^{\circledR}$ HTB-14 ${ }^{\mathrm{TM}}$ ), and $1321 \mathrm{~N} 1$ cell obtained from European Collection of Authenticated Cell Cultures (ECACC, catalog number 86030402).

All cell lines were cultured separately in $25-\mathrm{cm}^{2}$ culture flasks using DMEM supplemented with $10 \%$ FBS, $100 \mathrm{U} /$ $\mathrm{mL}$ penicillin, and $100 \mu \mathrm{g} / \mathrm{mL}$ streptomycin at $37^{\circ} \mathrm{C}$ in a humidified $5 \% \quad \mathrm{CO}_{2}$ atmosphere. The medium was changed every 2-3 days, and cells were sub-cultured when confluency reached $70-80 \%$ using $0.25 \%$ trypsin at $37^{\circ} \mathrm{C}$.

\section{Nucleic acids extraction}

When cells reached total confluence, they were washed with PBS, detached with $0.25 \%$ trypsin/EDTA (Gibco ${ }^{\mathrm{TM}}$ ), and suspended in PBS. DNA and RNA were extracted using the Wizard ${ }^{\circledR}$ Genomic DNA Purification kit (Promega Corporation, USA) and SV Total RNA Isolation System (Promega Corporation), respectively, according to the manufacturer's protocol.

DNA and RNA purity and integrity were assessed on the Agilent 2200 TapeStation (Agilent Technologies, USA) with D1000 ScreenTape (Agilent Technologies) and High Sensitivity RNA ScreenTape (Agilent Technologies) respectively, according to the manufacturer's protocol. Only samples with DNA Integrity Number (DIN) and RNA integrity $(\mathrm{RIN})>7$ were used for downstream analyses.

\section{Karyotype characterization}

For cytogenetic analyses, three different passages of the cell line were cultured and blocked by adding $100 \mu \mathrm{L}$ colcemid $(0.0016 \%)$. After incubation for one hour, cells were harvested with $0.05 \%$ trypsin, incubated with hypotonic solution $(0.075 \mathrm{KCl})$ for about $20 \mathrm{~min}$ at $37^{\circ} \mathrm{C}$, and fixed with 3:1 methanol/acetic acid. Afterwards, slides were prepared and submitted to standard Giemsa staining and G-banding using trypsin (4). The modal diploid number was defined after analysis of 50 metaphase plates. Chromosomes were arranged and described following the recommendations of the International System for Human Cytogenetic Nomenclature (ISCN).

\section{Array-based comparative genomic hybridization (aCGH) analysis}

Chromosomal imbalances analysis. Array-CGH (aCGH) experiments were performed on an Agilent microarray platform (Agilent Technologies) with a SurePrint G3 Cancer $\mathrm{CGH}+\mathrm{SNP}$ Microarray 4x180K slide (Agilent). Sample preparation, labelling, and microarray hybridization were performed according to the Agilent CGH Enzymatic Protocol 
version 7.5. Slides were scanned using the Agilent G2565CA scanner. Data were extracted with Feature Extraction software (v9.1 Agilent Technologies) and analyzed with Genomic Work Bench 11.0.1, Agilent Cytogenomics 5.0 and GeneSpring GX 14.5, as described elsewhere (5). The algorithm used was Aberration Detection Method 2 (ADM-2), applying the following filters: threshold=6; minimum number of probes in region=3; and Log2Ratio $>0.25$ and log2Ratio $<-0.25$ were defined as copy number gains and losses, respectively. Furthermore, we included the SNP Copy Number (confidence level 0.90), GC correction, diploid peak centralization, and LOH (threshold 6.0) in our analysis.

The ideogram showing the CNAs identified in AHOL1 genome was constructed using the PhenoGram online software (https://ritchielab.org/software/phenogram-down loads). The CNAs information of 1087 cancer cell lines from CellMiner and the Cancer Cell Line Encyclopedia (CCLE), 1987 human GBMs from TCGA database, stored in the cBioPortal for Cancer Genomics (accessed in June 2019) was used to explore the similarities with CNAs of AHOL1 cell line. Additionally, Candidate Cancer Gene Database (CCGD) was used to identify candidate cancer genes from CNAs of AHOL1 genome.

Gene expression microarray. Gene expression profiling analysis was performed using the Agilent Oligo Microarray Kit $8 \times 60 \mathrm{~K}$ according to the Agilent One-Color Microarraybased Gene Expression Analysis Protocol (Agilent Technologies). Data were extracted with Feature Extraction software (v9.1, Agilent Technologies) and analyzed with Gene Spring software GX 14.5 (Agilent Technologies). Raw data were normalized by robust multiarray average (RMA) quantile normalization analysis algorithm with the GeneSpring GX 14.5 software (Agilent Technologies) to generate CEL intensity files and the ratios were log2-transformed for multiple testing. We performed the quality control following diagnostic plots: principal component analysis (PCA), boxplots, Pearson's correlation, and MvA plots. All gene expression microarrays experiments were performed in triplicate.

Significantly differentially expressed genes (DEGs) were identified by using the mixed model analysis of variance with absolute fold-change values $\geqslant 2$. To reduce the false discovery rate (FDR), the $P$ value for significant differences was set to less than 0.05 .

Gene ontology enrichment analysis was performed using DAVID (Database for Annotation, Visualization and Integration Discovery) bioinformatics tools. Pathway analysis was performed with GeneSpring software GX 14.5 (Agilent Technologies) and the pathways were downloaded from the WikiPathways database (https://www. wikipathways.org/index.php/WikiPathways), BioCyc database (BioCyc.org), and KEGG database (https://www. genome.jp/kegg/pathway.html).

\section{Integrative analysis of CNAs and DEGs}

To identify the significant genes that exhibited CNA and gene expression alterations, we integrated the significant
CNAs and DEGs using GeneSpring software GX 14.5 (Agilent Technologies) as described elsewhere (5).

\section{Search for drugs targeting CNAs}

The Drug-Gene Interaction Database (DGldb; https:// www.dgidb.org/) was used to search potential drugs targeting CNAs. This database provides gene druggability information from different databases (such as Therapeutic Target database, DrugBank, Pharmacogenomics Knowledge database, papers, and web resources).

\section{Reverse transcription qPCR (RT-qPCR)}

For the cDNA synthesis, we used the GoScript ${ }^{\mathrm{TM}}$ Reverse Transcription System (Promega Corporation) following the manufacturer's instructions. Real time PCR (qPCR) was performed as described by Ferreira et al. (6), using GoTaq ${ }^{\mathbb{R}}$ Probe qPCR Master Mix (Promega Corp.). All reactions were carried out in triplicate in 96-well PCR plates, using CFX96 Touch $^{\mathrm{TM}}$ Real-Time PCR Detection System (Bio-Rad, USA). Data analysis was performed using the Bio-Rad CFX Manager ${ }^{\mathrm{TM}} 3.1$ software (Bio-Rad). Following the MIQE guidelines, the expression levels were normalized using TBP and GAPDH in non-neoplastic samples. The relative gene expression was calculated using the $2^{-\triangle \Delta C T}$ formula $(P<0.05)$.

The expression of the genes ANOS1, ETV1, XPNPEP2, and $P C D H 11 Y$ was quantitated using Taqman ${ }^{\circledR}$ gene expression assays (Applied Biosystems, USA) (Table 1).

\section{Results}

\section{Cytogenetic characterization}

We obtained and analyzed 50 chromosome spreads of the AHOL1 cell line. No normal karyotype was found in any of the metaphases analyzed. All metaphases were hyperdiploid, with chromosome number varying from $2 n=51$ to $2 n=59$, with a modal number of 54 and 57 , both showing a frequency of $26 \%$ each (13/50). G-banding analysis confirmed the occurrence of both numerical and structural rearrangements, with loss/gain of chromosomes or chromosome arms, and fission of some pairs. Most numerical rearrangements involved gain of chromosomes of group $\mathrm{E}$. Despite the fact of AHOL1 has been obtained from a male patient, the chromosome $Y$ was missing in all the metaphases analyzed by G-band. Additionally, most cells gained an extra copy of chromosome $\mathrm{X}$. A representative karyotype is shown in Figure 1. An overall idea of common quantitative alterations were detected by aCGH.

\section{CNA profiling of AHOL1 cell line}

A global view of the AHOL1 CNAs composition was generated using the a-CGH results. A total of 19 CNAs (17 gains and 2 losses) were identified, ranging from $0.28 \mathrm{Mb}$ to $93 \mathrm{Mb}$. A full list of the CNAs and their corresponding chromosome localization, cytobands, type of alteration, 
Table 1. Targets and housekeeping genes used in this study.

\begin{tabular}{lcc}
\hline Official gene symbol $^{*}$ & Official full name $^{*}$ & Assay ID \\
\hline GAPDH & Glyceraldehyde-3-phosphate dehydrogenase & Hs02786624_g1 \\
TBP & TATA-box binding protein & Hs00427620_m1 \\
ANOS1 & Anosmin 1 & Hs01085107_m1 \\
ETV1 & ETS variant 1 & Hs00951951_m1 \\
XPNPEP2 & X-prolyl aminopeptidase 2 & Hs00950918_m1 \\
PCDH11Y & Protocadherin 11 Y-linked & Hs06651077_g1 \\
\hline
\end{tabular}

*Official symbols and names of the genes were based on HUGO gene nomenclature committee (HGNC).

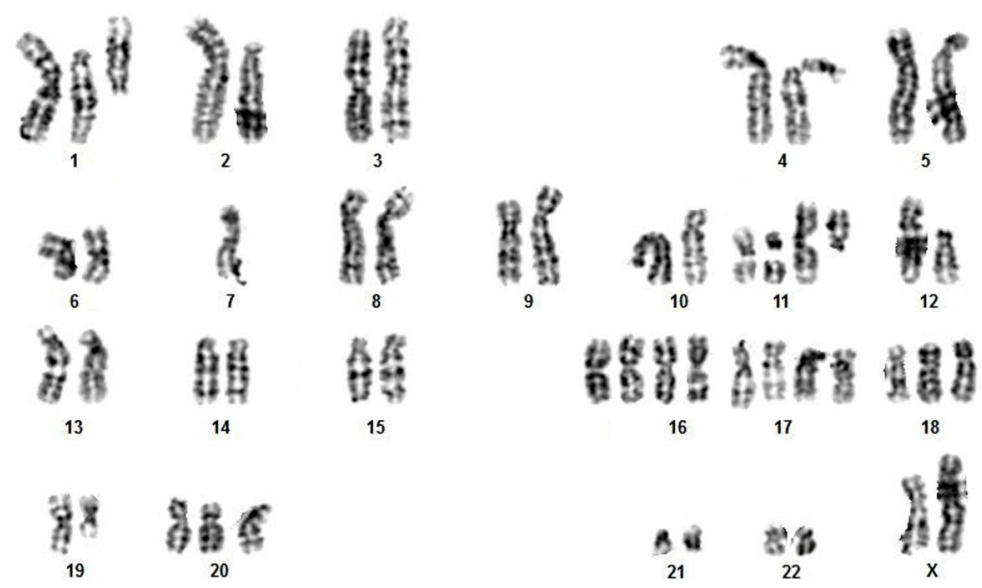

Figure 1. A representative G-banded metaphase of AHOL1 cell line, with $2 n=54, X X,+\operatorname{der}(1),+\operatorname{der}(1),+\operatorname{der}(2), \operatorname{del}(6 q), \operatorname{del}(6 q),-7$, $+\operatorname{der}(11),+\operatorname{der}(11), \operatorname{del}(11 q), \operatorname{del}(12 p),+16,+16,+17,+17,+18, \operatorname{del}(19 q),+\operatorname{der} 20$.

$\mathrm{P}$ value, and genes are provided in Supplementary Table S1. Copy number gains were located at chromosomes $4,6,7,8,9,10,11,14,17$, and 19, while losses were at chromosome 15 (Figure 2; Supplementary Table S1). Whole chromosome gains and losses were observed at chromosomes $\mathrm{X}$ and $\mathrm{Y}$, respectively.

The highest number of gains was found at chr $X$ at q11.1-q28 (93,148.679 kb - with 3016 segments), p22.33p11.1 (55,799.123 kb - with 1745 segments), and q21.1q21.33 $(13,545,889 \mathrm{~kb})$ positions. The second highest number of gains was documented at chromosome 4 (cytoband p15.1-p14) corresponding to 7,800 kb, followed by chromosome 8 (cytoband q23.2-q23.3) corresponding to $5,104 \mathrm{~kb}$. Additionally, we identified that the highest frequency of losses occurred on the $Y$ chromosome at q11.21-q12 (45,327.039 kb) and p11.31-p11.2 (7,119.122 $\mathrm{kb})$ positions (Figure 2).

We further explored whether AHOL1 CNAs were recurrent in cancer cell lines from CellMiner and CCLE databases ( $\mathrm{N}=1087$ cell lines) ( $\geqslant 10 \%$ of frequency). Indeed, in silico analysis revealed that thirty-one genes were covered by CNAs in most cancer cell lines (Supplementary Table S2). In addition, by performing Ingenuity

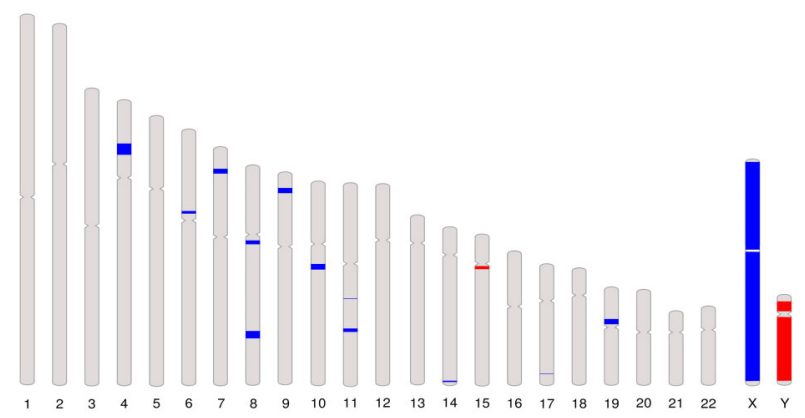

Figure 2. Representative copy number alteration (ideogram) showing gains (blue), and losses (red) in the AHOL1 genome.

Pathway Analysis (IPA), a total of 240 cancer-related genes, 15 of them exclusively related with brain cancer (Supplementary Table S3) were revealed. Also, we analyzed whether CNAs detected in AHOL1 were frequently found across primary GBM tumors from the TCGA database. Interestingly, the vast majority of genes covered by CNAs were commonly altered across several primary GBM tumors. 
We also conducted an analysis to detect the main altered pathways affected by CNAs in the AHOL1 genome. Amplifications affected 60 pathways, such as putrescine degradation III, melatonin degradation II, and leucine degradation pathways, while losses had no impact in any pathway (Supplementary Table S4).

\section{AHOL1 is genomically similar to other human GBM cell lines}

Considering that the AHOL1 cell line was established from a GBM patient, we expected it to share common CNAs with commercial GBM cell lines (U87MG, U343, and $1321 \mathrm{~N} 1$ ). As shown in Figure 3, our results indicated the existence of a common genomic signature between AHOL1 and commercial GBM cell lines (1321N1, U343, and U87), thus confirming its GBM identity.

\section{Drugs targeting CNAs}

Potential drugs targeting CNAs were investigated using the DGIdb database. Nine druggable genes were identified, including HCRTR2 (hypocretin receptor 2), ETV1 (ETS variant 1), PTPRD (protein tyrosine phosphatase, receptor type D), PRKX (protein kinase X-linked), STS (steroid sulfatase), RPS6KA6 (ribosomal protein S6 kinase A6), ZFY (zinc finger protein Y-linked), USP9Y (ubiquitin specific peptidase 9 Y-linked), and KDM5D (lysine demethylase 5D) (Table 2).

\section{Transcriptome characterization of AHOL1 cell line}

In total, we identified 1,837 DEGs. Among these, 713 genes were upregulated, whereas 1,124 genes were downregulated ( $F C \geqslant 2$ and $P<0.05$ ) (Table 3 ). A full list of differentially expressed genes and their corresponding

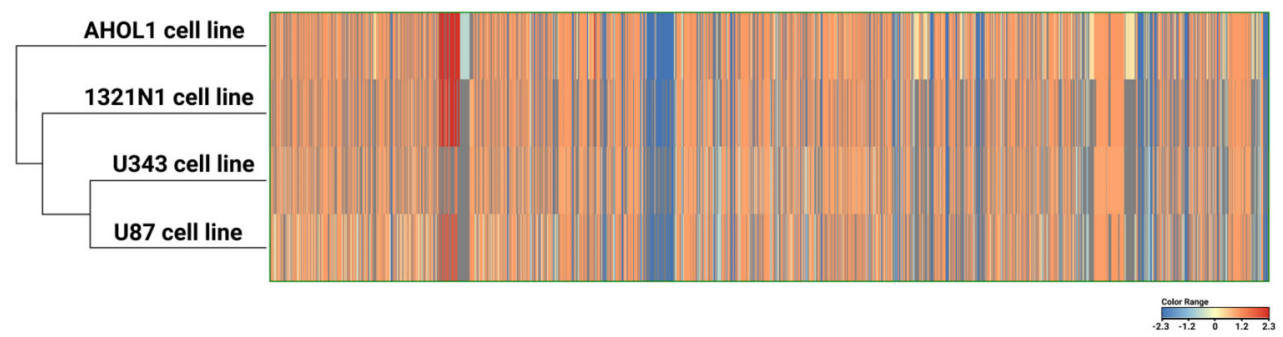

Figure 3. Heatmap of all copy number alterations present in each cell line (AHOL1, 1321N1, U343, and U87).

Table 2. Druggable genes of the AHOL1 cell line. Information of each gene regarding their chromosomal location, cytoband, size (kb), type of alteration found (gain or loss), drugs, and the type of interaction of each drug is provided below.

\begin{tabular}{|c|c|c|c|c|c|c|}
\hline Chromosome & Cytoband & Size (Kb) & Variant type & Gene & Drug & Interaction type \\
\hline \multirow[t]{4}{*}{6} & $\mathrm{p} 12.1$ & 1,752 & Gain & HCRTR2 & Suvorexant & Antagonist \\
\hline & & & & & SB-649868 & Antagonist \\
\hline & & & & & Lemborexant & Antagonist \\
\hline & & & & & Almorexant & $\mathrm{n} / \mathrm{a}$ \\
\hline 7 & p21.3 - p21.2 & 3,393 & Gain & ETV1 & Trametinib & $\mathrm{n} / \mathrm{a}$ \\
\hline \multirow[t]{3}{*}{10} & $\mathrm{q} 21.1$ & 3,864 & Gain & PTPRD & Cucurbitacin & $\mathrm{n} / \mathrm{a}$ \\
\hline & & & & & Teprotumumab & n/a \\
\hline & & & & & Cixutumumab & $\mathrm{n} / \mathrm{a}$ \\
\hline \multirow[t]{7}{*}{$x$} & p22.33 - p11.1 & 55,799 & Gain & PRKX & GSK-690693 & Inhibitor \\
\hline & & & Gain & STS & Estrone & $\mathrm{n} / \mathrm{a}$ \\
\hline & & & & & Danazol & $\mathrm{n} / \mathrm{a}$ \\
\hline & & & & & Triptorelin & $\mathrm{n} / \mathrm{a}$ \\
\hline & & & & & Penicillamine & $\mathrm{n} / \mathrm{a}$ \\
\hline & & & & & Progesterone & $\mathrm{n} / \mathrm{a}$ \\
\hline & & & & & STS & Inhibitor \\
\hline \multirow[t]{2}{*}{$x$} & q21.1 - q21.33 & 13,545 & Gain & RPS6KA6 & AT-9283 & Inhibitor \\
\hline & & & & & Chembl573107 & Inhibitor \\
\hline$Y$ & p11.31 - p11.2 & 7,119 & Loss & ZFY & Chembl383208 & \\
\hline \multirow[t]{2}{*}{$Y$} & $\mathrm{q} 11.21$ - q12 & 45,327 & Loss & USP9Y & Testosterone & $n / a$ \\
\hline & & & & KDM5D & Ascorbate & $\mathrm{n} / \mathrm{a}$ \\
\hline
\end{tabular}

n/a: not available. 
fold changes in expression and $P$ values are provided in Supplementary Table S5. Ret Finger Protein-like 4A-like 1 (RFPL4AL1) was the most upregulated (FC: 55.87), and lincRNA Inc-CHIC1-2:1 the most downregulated mRNA (FC: -65.82) (Table 3).

To better understand the biological processes associated with DEGs, Gene Ontology (GO) analysis was conducted. The majority of DEGs were distributed into four GO categories: biological process, molecular function, cellular component, and protein class (Figure 4).
A full list of GO terms is provided in Supplementary Table S6.

In the biological process category, the most enriched terms were related to biological regulation and metabolic process (Figure 4A). Binding and catalytic activity mostly accounted for terms related to the molecular function category (Figure 4B). Within the cellular component category, the GO term with the highest level of significance was cell, followed by membrane and organelle (Figure $4 \mathrm{C}$ ). Finally, in the protein class category, the

Table 3. Characteristics of the top 20 differentially expressed mRNAs in AHOL1 cell line sorted by fold change (fold change $\geqslant 2$ and $\mathrm{P}<0.05)$.

\begin{tabular}{|c|c|c|c|c|}
\hline & Gene symbol & Chromosome & Description & Fold change \\
\hline \multirow[t]{10}{*}{ Upregulated } & RFPL4AL1 & chr19 & Ret Finger Protein-like 4A-like 1 & 55.87 \\
\hline & Inc-WDR5-2 & chr9 & lincRNA & 27.04 \\
\hline & ERICH1-AS1 & chr8 & IncRNA & 26.70 \\
\hline & LRRN4 & chr7 & Leucine Rich Repeat Neuronal 4 & 21.44 \\
\hline & CMTR1 & chr6 & Cap Methyltransferase 1 & 21.38 \\
\hline & XLOC_I2_012743 & chr6 & IncRNA & 11.53 \\
\hline & LINC01297 & chr14 & lincRNA & 10.86 \\
\hline & KDM4E & chr11 & Lysine demethylase 4E & 9.08 \\
\hline & Inc-RBPJ-1: 1 & chr4 & lincRNA & 8.59 \\
\hline & Inc-FBXO25-5: 4 & chr8 & lincRNA & 6.21 \\
\hline \multirow{10}{*}{ Downregulated } & Inc-CHIC1-2: 1 & $\operatorname{chr} X$ & lincRNA & -65.82 \\
\hline & SHANK3 & chr22 & SH3 and multiple ankyrin repeat domains 3 & -36.71 \\
\hline & SNORD114-3 & chr14 & Small nucleolar RNA, C/D box $114-3$ & -18.68 \\
\hline & LOC403323 & chr9 & Uncharacterized LOC403323 & -12.19 \\
\hline & ZFP57 & chr6 & ZFP57 zinc finger protein & -10.78 \\
\hline & CYP11A1 & chr15 & Cytochrome P450 family 11 subfamily A member 1 & -9.88 \\
\hline & DSP & chr6 & Desmoplakin & -9.16 \\
\hline & PLVAP & chr19 & Plasmalemma vesicle associated protein & -8.89 \\
\hline & C4BPA & chr1 & Complement component 4 binding protein alpha & -8.72 \\
\hline & Inc-ZNF100-2 & chr19 & IncRNA & -7.92 \\
\hline
\end{tabular}

A

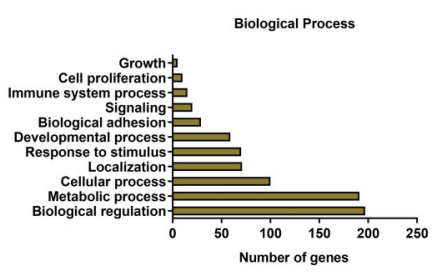

C

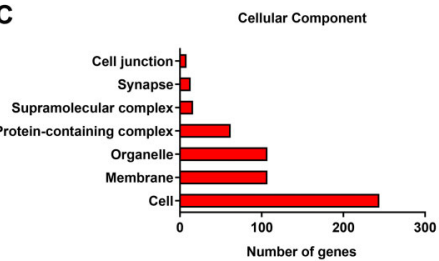

B

Translation regulator activity -1 Structural molecule activity Molecular function regulatorTranscription activity Transporter activity Molecular transducer activity Catalytic activity $=$

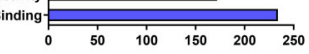
Number of genes

D Protein Class

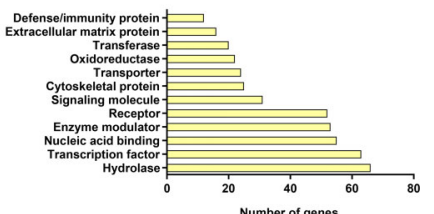

Figure 4. Gene Ontology (GO) functional annotations for differentially expressed genes (DEGs) of the AHOL1 cell line. Bar graphs show four GO categories: (A) Biological processes, (B) Molecular functions, (C) Cellular components, and (D) Protein classes. The X-axis represents the number of DEGs and the $\mathrm{Y}$-axis shows the $\mathrm{GO}$ terms of each category (fold change $\geqslant 2$ and $\mathrm{P}<0.05$ ). 
Table 4. Pathway analysis of differentially expressed genes from AHOL1 cell line.

\begin{tabular}{lc}
\hline Pathways & P value \\
\hline Upregulated & \\
Trans, trans-farnesyl diphosphate biosynthesis & 0.0262 \\
Superpathway of geranylgeranyldiphosphate biosynthesis I (via mevalonate) & 0.0391 \\
Mevalonate pathway & 0.0391 \\
IL1 & 0.0397 \\
4-hydroxy-2-nonenal detoxification & 0.0414 \\
Glycogenolysis & 0.0311 \\
mRNA capping & 0.0417 \\
Downregulated & \\
Pathways in cancer & 0.0091 \\
C20 prostanoid biosynthesis & 0.0169 \\
Plasmalogen degradation & 0.0229 \\
Rapoport-Luebering glycolytic shunt & 0.0229 \\
Pyridoxal 5'-phosphate salvage & 0.0229 \\
Choline degradation & 0.0229 \\
\hline
\end{tabular}

Pathways were selected according to the $P$ value (fold change $\geqslant 2$ and $P<0.05$ ).

terms hydrolase and transcription factor exhibited the highest significance (Figure 4D).

Pathway analysis was performed to investigate the biological significance of these DEGs. Thirteen pathways were significantly affected in the AHOL1 cell line, such as mevalonate, IL-1, glycogenolysis, and mRNA capping pathways in cancer (Table 4).

\section{AHOL1 cell line shares several DEGs with other commercial GBM cell lines}

To determine the number of genes shared between AHOL1 and the commercial GBM cell lines, Venn diagrams were created. Our results showed that AHOL1 shared several transcripts with the commercial GBM cell lines.

Analysis of transcriptomes highlighted that the AHOL1 cell line has several changes common to the different GBM commercial cell lines. All cell lines had shared 756 upregulated and 281 downregulated genes (Figure 5).

\section{Integrative analysis of CNA and gene expression profiling}

To explore and compare how CNAs affect AHOL1 transcriptional program, we performed an integrative analysis of CNAs with DEGs. Most of DEGs showed no positive correlation with CNAs. Only 57 genes displayed significant CNA-DEGs correlation in the AHOL1 cell line. A full list of these overlapping genes is found in Supplementary Table S7. Twenty-four genes showed a positive association, while 33 genes presented an inverse association (Table 5).

To further understand the biological function of these 57 overlapping genes, they were submitted to functional annotation and classification analysis. Most of these genes were enriched for metabolic processes, biological regulation, and cellular processes in the biological category (Figure 6A). For the molecular function category, the
A

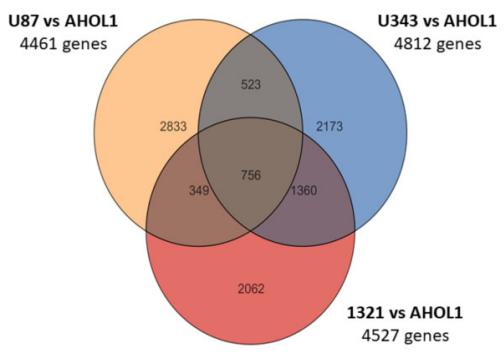

B

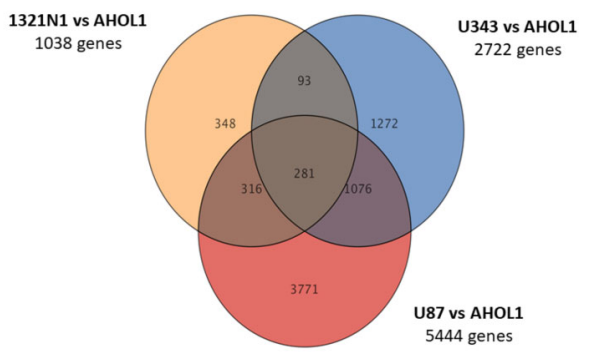

Figure 5. Venn diagram showing the number of genes upregulated (A) and downregulated (B) in all glioblastoma cell lines.

GO terms with the highest levels were catalytic activity, binding, and transcription activity (Figure 6B). Within the cellular component category, cell was the highest term, followed by membrane and organelle terms (Figure 6C). Of note, in the protein class category, the terms nucleic acid binding and transcription factor exhibited the highest frequencies (Figure 6D).

Next, we performed separately GO functional annotation of each group shown in Table 5. As shown in 
Supplementary Table S8, most genes covered by gains whose expression was upregulated mainly affected the nucleus (Cellular Component), provoking changes in the DNA-binding transcription factor (TF) activity (Molecular Function) of the helix-turn-helix and zinc finger TFs (Protein Class), which consequently had an effect on the cell development and cell differentiation (Biological process).

Genes covered by gains and whose expression was downregulated mostly affected the membranes (Cellular Component), especially disturbing the hydrolase activity (Molecular Function), modifying the cell communication, signal transduction, cellular response to stimulus, and cellular metabolic process (Biological Process). Furthermore, genes covered by losses and whose expression was upregulated essentially affected the cytoplasm (Cellular Component), altering the RNA binding (Molecular Function) of the ribosomal proteins and translation factors (Protein Class) and influencing the translational elongation and termination (Biological Process). Finally, $\mathrm{PCDH} 11 \mathrm{Y}$ loss and down-regulation affected plasma membrane, by altering the cell adhesion and inducing changes in the calcium ion binding (Supplementary Table S8)

Table 5. Overlapping genes identified after integration of copy number alteration (CNAs) and gene expression data of AHOL 1 cell line.

\begin{tabular}{lcc}
\hline CNA & Gene expression & Overlapping genes \\
\hline Gain & Upregulated & 13 \\
Gain & Downregulated & 32 \\
Loss & Upregulated & 11 \\
Loss & Downregulated & 1 \\
Total & & 57 \\
\hline
\end{tabular}
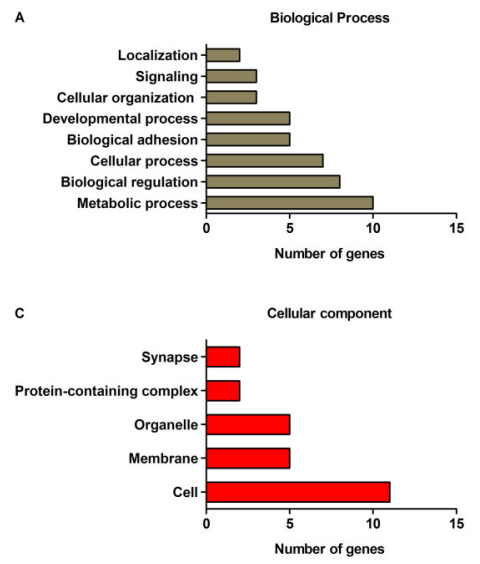

We also conducted a global analysis with all 57 genes from the DEGs-CNAs dataset in order to detect the main altered pathways from AHOL1. Signaling pathways via Receptor-type tyrosine protein phosphatases, Cadherin, Wnt, FGFR1, and Akt, as well as metabolism of proteins were statistically significant.

\section{qRT-PCR validation of the microarray results}

To validate the DEGs-CNAs dataset generated by microarray, we selected four genes related to cancer. Three of them (ANOS1, ETV1, and XPNPEP2) were upregulated via copy number gain and one gene ( $P C D H 11 Y)$ was downregulated via copy number loss. Transcription levels of selected genes are shown in Figure 7.

Gene expression showed that ANOS1, ETV1, and $X P N P E P 2$ were upregulated in AHOL1 relative to normal brain tissue fragments (2-fold; $>4$ fold; 2.7-fold, respectively), which is in line with the microarray data (Figure 7 and Supplementary Table S5). Of note, $P C D H 11 Y$ was 2.3-fold downregulated in AHOL1 relative to normal brain tissues, in agreement with the microarray data (Figure 7D; Supplementary Table S5).

\section{Discussion}

Recent data provided by The Cancer Genome Atlas (TCGA) consortium have shown great genetic and epigenetic diversity among GBM tumors (1). The elucidation of the biological mechanisms and the complexity behind this diversity is a central challenge for achieving precision medicine in GBM subtypes. For this reason, the availability of a large number of molecularly well-characterized GBM cell lines may have a high impact on understanding the complex biology of this tumor, thus contributing to identification of new therapeutic targets.

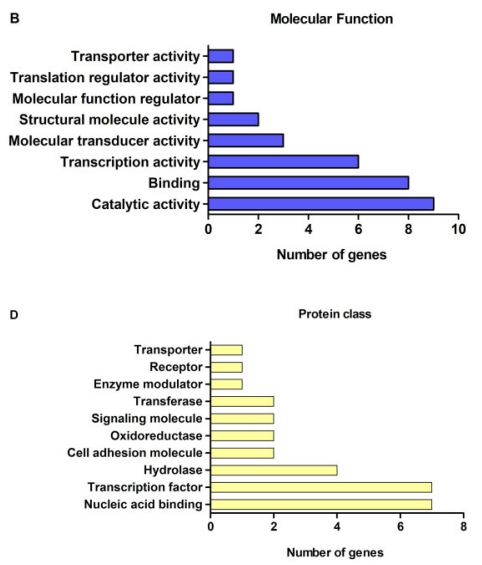

Figure 6. Functional classification of integrated differentially expressed genes (DEGs) copy number alterations (CNAs) data from AHOL1 cell line using Gene Ontology (GO) analysis. Bar graphs show four independent GO information categories: (A) Biological processes, (B) Molecular functions, (C) Cellular components, and (D) Protein classes. The Y-axis represents the number of genes of integrated DEGs-CNAs data and the $X$-axis shows the GO terms for each category $(P<0.05)$. 

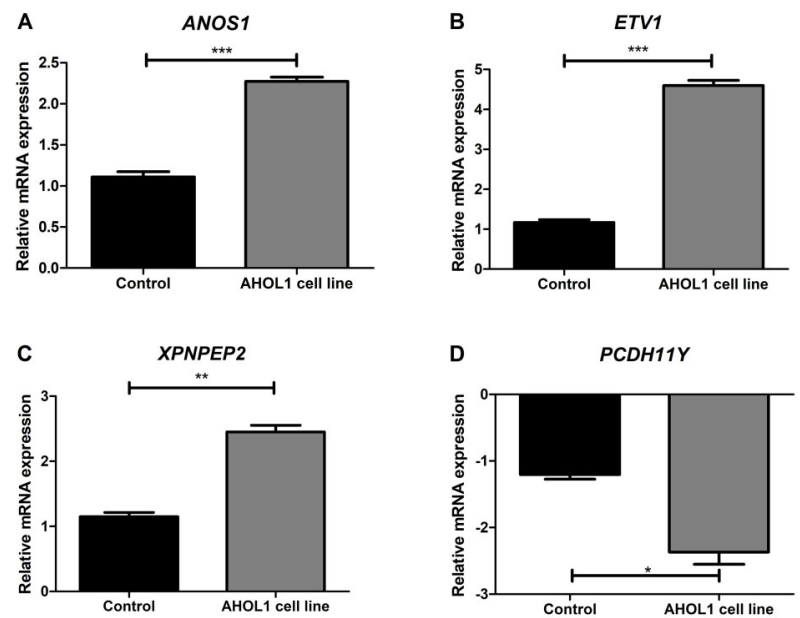

Figure 7. Validation of the microarray data by qRT-PCR. qRTPCR was performed for ANOS1 (A), ETV1 (B), XPNPEP2 (C), and $P C D H 11 Y$ (D) genes. Data are reported as fold change in mRNA expression and compared with a tissue fragment from normal brain as a control. Each bar represents means $\pm S D$ values for each gene with three technical replicates. Statistical significance was determined using Student's $t$-test: ${ }^{*} \mathrm{P}<0.05$; ${ }^{* *} \mathrm{P}<0.01 ;{ }^{* *} \mathrm{P}<0.001$

As an attempt to better understand the complexity of the GBM biology and the phenomena that pervade the aspects of its intricate mechanisms, we provide the first comprehensive cytogenomic profile of the human glioblastoma cell line AHOL1. This cell line showed a hyperdiploid karyotype, and clearly exhibited recurrent CNAs consistent with those observed in primary GBM tumors from TCGA database, CellMiner, and Cancer Cell Line Encyclopedia, as well as the commercial GBM cell lines (1321N1, U343, and U87) used in this study and other reports $(7,8)$. Among these, the most prominent CNAs were documented at $X$ - (gain of entire chromosome) and $Y$-chromosomes (loss of entire chromosome), in line with previous cytogenetics and molecular reports for GBM tumors (9).

Chromosomal rearrangements are common in cancer cells, and it is proposed that they influence cancer development, progression, metastasis, and drug resistance due to possible alteration in gene expression, not only by gain/ loss, but also by translocations. Thus, a better understanding of cancer-causing chromosomal rearrangements may enable the development of novel anticancer protocols (10). In this sense, although many functional aspects of CNAs at chr $X$ and chr $Y$ have not yet been extensively studied in cancer, a preliminary study suggested that high expression of SPIN4 and ASB12 was correlated with amplification at Xq11 in GBM (11), thus affecting several important biological processes, such as mitosis, Wnt signaling pathway, $\mathrm{H} 3 \mathrm{~K} 4 \mathrm{me} 3$, and post-translational protein modifications. Recent reports also support that the $\mathrm{X}$ chromosome gain plays a prominent role in the neoplastic transformation of breast cancer (12), chronic neutrophilic leukemia (13), non-Hodgkin lymphoma (14), renal cell carcinoma (15), and prostate cancer (16).

Massive amounts of cancer genomics data generated from next-generation sequencing have motivated investigators to develop novel computational approaches for the identification of new druggable genes, which can be used as therapeutic targets in precision cancer medicine. Our AHOL1 genomic data showed that nine genes (HCRTR2, ETV1, PTPRD, PRKX, STS, RPS6KA6, ZFY, USP9Y, and $K D M 5 D)$ are known as potential druggable anticancer targets, and some of them could be druggable vulnerabilities in some subtypes of GBM (17). Together, these studies have highlighted the great potential of AHOL 1 cell line for in vitro studies.

The association between chromosome rearrangements, including CNAs, and gene expression profiling suggested the identified alterations could contribute to the expression of some but not all genes. In some cases, expression changes were inconsistent with the CNAs. This might be influenced by other factors that contribute to gene expression variation, such as epigenetics changes, non-coding RNA regulation, gene mutation, and altered expression of TFs (18). This apparent discrepancy between copy number status and gene expression has also been observed in other cancers (19).

In this study, pathway enrichment of CNA-driven DEGs indicated significant changes in six pathways, where the majority of genes were prominently involved in the signaling by tyrosine phosphorylation. Tyrosine phosphorylation plays an important role in regulating cellular function and is a central feature in signaling cascades involved in oncogenesis. This process is coordinately controlled by protein tyrosine phosphatase (PTPs) and protein tyrosine kinases (PTKs), which are altered in a variety of human cancers, including GBMs (20). Among the tyrosine phosphatases, the tumor suppressor PTPRD is one of those often inactivated by deletion ( $>50 \%$ of cases), whose loss of expression promotes gliomagenesis through aberrant STAT3 activation (21) and is related with poor prognosis in GBM patients. Our observations, therefore, suggested that this gene was amplified and downregulated in AHOL1. Perhaps, this gene might be epigenetically silenced in AHOL1, once GBMs that do not harbor loss of PTPRD have this gene inactivated by its promoter hypermethylation (22).

Assuming that CNA-DEG integrated data have been shown to be an efficient approach to identify genes covered by altered CNAs that directly change their expression levels, not all genes of our integrated dataset were cancerrelated. In order to explore only genes related to cancer, we selected four genes, including three that were upregulated via copy number gain (ANOS1, ETV1, and XPNPEP2) and one gene $(P C D H 11 Y)$ that was downregulated via copy number loss. 
ANOS1, also known as $K A L 1$, encodes anosmin-1, an extracellular matrix (ECM)-associated protein that plays essential roles in neural cell adhesion and axonal migration. The upregulation of this gene in the AHOL1 cell line was consistent with the results from Choy et al. (23) for GBM and low-grade astrocytic tumors, as they found that anosmin-1 enhanced cell motility and proliferation in GBM cell lines. The overexpression of ANOS1 is also related to development and metastasis of colorectal cancer and its expression is closely related to the overall survival rate of patients (24).

Previous studies have shown that the oncogene ETV1 (ETS variant 1 - member of the ETS family of transcription factors) contributes to neoplastic transformation of prostate cancer (25), breast cancer (26), esophageal adenocarcinoma (27), gastrointestinal stromal tumors (28), cranial germinomas (29), gastric cancer (30), melanoma brain metastases (31), and melanoma (32). In GBMs, high expression of ETV1 may be a central downstream effector of chromosome 7 gain (33). Additionally, ETV1 is likely to be methylated in $\mathrm{CIC}$ wild-type, IDH-mutated, 1p/19qcodeleted gliomas (34), and fused with DGKB (35) in pediatric high-grade gliomas, acting as an oncogenic driver. In line with these results, we here demonstrated that the up-regulation of ETV1 was due to a chr7 gain in AHOL1 genome. Thus, the high prevalence of upregulated ETV1 together with chromosome $7 \mathrm{p}$ gain in GBMs may be new targets for efficacious therapies to improve outcomes of patients.

$X P N P E P 2$ is a membrane-bound aminopeptidase $P$ member of the 'pita bread fold' family, which catalyzes the removal of the penultimate prolyl residue from $\mathrm{N}$-termini of peptides. Although it is known that this aminopeptidase activates growth factors, hormones, coagulants, toxins, cytokines, and neurotransmitters, the role of XPNPEP2 in cancer is still unknown. It is known that XPNPEP2 is overexpressed in cervical cancer, promoting cell invasion and migration without affecting cell proliferation and apoptosis (36). Our gene expression results corroborated these findings and point out that XPNPEP2 can be upregulated in GBM.

Protocadherins constitute the largest subfamily of cadherins in the genome. Protocadherins genes are

\section{References}

1. Louis DN, Perry A, Reifenberger G, von Deimling A, Figarella-Branger D, Cavenee WK, et al. The 2016 World Health Organization Classification of Tumors of the Central Nervous System: a summary. Acta Neuropathol 2016; 131: 803-820, doi: 10.1007/s00401-016-1545-1.

2. Korshunov A, Casalini B, Chavez L, Hielscher T, Sill M, Ryzhova M, et al. Integrated molecular characterization of IDH-mutant glioblastomas. Neuropathol Appl Neurobiol 2019; 45: 108-118, doi: 10.1111/nan.12523. predominantly expressed in the nervous system, acting in crucial functions associated with formation, maintenance, and integrity of the neural circuit (37). Recently, they have been in the spotlight for their roles in cancer (38). Protocadherin-11Y (also named as PCDH11Y) is a proto-oncogene candidate exclusively found in man and its transcription occurs mainly in the brain (37). This gene is upregulated in prostate carcinoma (39), inducing neuroendocrine transdifferentiation through activation of the wnt signaling (40). To the best of our knowledge, this is the first study showing that $P C D H 11 Y$ was downregulated in GBM, contradicting the results found elsewhere (39).

In summary, we described for the first time the genome and transcriptome of the new human cell line AHOL1, established from a GBM patient. Here, we revealed that this cell line harbored a genomic alteration spectrum similar to what is observed in commercial GBM cell lines (U87MG, U343, and 1321N1) and GBMs from the TCGA database, and some of these CNAs can be targeted by drugs, suggesting that this new cell line is a suitable model system for understanding the molecular characteristics of human GBM tumorigenesis.

\section{Supplementary Material}

Click to view [zip].

\section{Acknowledgments}

This research was funded by Conselho Nacional de Desenvolvimento Científico e Tecnológico (CNPq) (Grant Number: 455078/2014-9) and Evandro Chagas Institute. The authors are grateful to Instituto Evandro Chagas (Brazil), Pró-Reitoria de Pesquisa e Pós-Graduação (PROPESP/UFPA, Brazil), and CNPq for financial and technical support; to the Hospital Ophir Loyola (Brazil) and the patient's family for the consent to participate in this project; to Dr. Rommel Rodriguez Burbano for providing the AHOL1 cell line used in this study; to Coordenação de Aperfeiçoamento de Pessoal de Nível Superior (CAPES) for W.A.S. Ferreira's PhD fellowship.

3. Pereira EL, Lima PD, Khayat AS, Bahia MO, Bezerra FS, Andrade-Neto $\mathrm{M}$, et al. Inhibitory effect of pisosterol on human glioblastoma cell lines with C-MYC amplification. J Appl Toxicol 2011; 31: 554-560, doi: 10.1002/jat.1596.

4. Seabright M. A rapid banding technique for human chromosomes. Lancet 1971; 2: 971-972, doi: 10.1016/S0140-6736 (71) $90287-X$.

5. Liu D, Xu X, Wen J, Xie L, Zhang J, Shen Y, et al. Integrated genome-wide analysis of gene expression and DNA copy 
number variations highlights stem cell-related pathways in small cell esophageal carcinoma. Stem Cells Int 2018; 2018: 3481783, doi: 10.1155/2018/3481783.

6. Ferreira WA, Araujo MD, Anselmo NP, de Oliveira EH, Brito JR, Burbano RR, et al. Expression analysis of genes involved in the RB/E2F pathway in astrocytic tumors. PLoS One 2015; 10: e0137259, doi: 10.1371/journal.pone.0137259.

7. Cohen A, Sato M, Aldape K, Mason CC, Alfaro-Munoz K, Heathcock L, et al. DNA copy number analysis of Grade II-III and Grade IV gliomas reveals differences in molecular ontogeny including chromothripsis associated with IDH mutation status. Acta Neuropathol Commun 2015; 3: 34, doi: 10.1186/s40478-015-0213-3.

8. Burbulis IE, Wierman MB, Wolpert M, Haakenson M, Lopes $\mathrm{MB}$, Schiff $\mathrm{D}$, et al. Improved molecular karyotyping in glioblastoma. Mutat Res 2018; 811: 16-26, doi: 10.1016/j. mrfmmm.2018.06.002.

9. Kim DH, Mohapatra G, Bollen A, Waldman FM, Feuerstein BG. Chromosomal abnormalities in glioblastoma multiforme tumors and glioma cell lines detected by comparative genomic hybridization. Int J Cancer 1995; 60: 812-819, doi: 10.1002/ijc.2910600615.

10. Hasty $P$, Montagna C. Chromosomal rearrangements in cancer: detection and potential causal mechanisms. Mol Cell Oncol 2014; 1: e29904, doi: 10.4161/mco.29904.

11. Canello T, Shahar T, Zelikovitch B, Mordechai A, Lossos A, Lavon I. GENO-23* Amplification of chromosome xq11. 1xq11. 2 might play a role in glioblastoma. Neuro Oncol 2015; 17: v96, doi: 10.1093/neuonc/nov215.23.

12. Di Oto E, Biserni GB, Varga Z, Morandi L, Cucchi MC, Masetti $R$, et al. $X$ chromosome gain is related to increased androgen receptor expression in male breast cancer. Virchows Arch 2018; 473: 155-163, doi: 10.1007/s00428018-2377-2.

13. Yamamoto K, Nagata K, Kida A, Hamaguchi H. Acquired gain of an $X$ chromosome as the sole abnormality in the blast crisis of chronic neutrophilic leukemia. Cancer Genet Cytogenet 2002; 134: 84-87, doi: 10.1016/S0165-4608(01) 00603-3.

14. McDonald HL, Gascoyne RD, Horsman D, Brown CJ. Involvement of the $\mathrm{X}$ chromosome in non-Hodgkin lymphoma Genes Chromosomes Cancer 2000; 28: 246-257, doi: 10.1002/1098-2264(200007)28:3<246::AID-GCC2>3. $0 . \mathrm{CO} ; 2-0$.

15. Kuroda N, Shiotsu T, Hes O, Michal M, Shuin T, Lee GH. Acquired cystic disease-associated renal cell carcinoma with gain of chromosomes 3,7 , and 16, gain of chromosome $\mathrm{X}$, and loss of chromosome Y. Med Mol Morphol 2010; 43: 231-234, doi: 10.1007/s00795-009-0465-8.

16. Qu X, Jeldres C, Glaskova L, Friedman C, Schroeder S, Nelson PS, et al. Identification of combinatorial genomic abnormalities associated with prostate cancer early recurrence. J Mol Diagn 2016; 18: 215-224, doi: 10.1016/ j.jmoldx.2015.10.001.

17. Nguyen HS, Shabani S, Awad AJ, Kaushal M, Doan N. Molecular markers of therapy-resistant glioblastoma and potential strategy to combat resistance. Int $J \mathrm{Mol} \mathrm{Sci}$ 2018;19: 1765, doi: 10.3390/ijms19061765.

18. Gamazon ER, Stranger BE. The impact of human copy number variation on gene expression. Brief Funct Genomics 2015; 14: 352-357, doi: 10.1093/bfgp/elv017.
19. Yan D, Yi S, Chiu WC, Qin LG, Kin WH, Kwok Hung CT, et al. Integrated analysis of chromosome copy number variation and gene expression in cervical carcinoma. Oncotarget 2017; 8: 108912-108922, doi: 10.18632/on cotarget.22403.

20. Hamaoka $\mathrm{Y}$, Negishi $\mathrm{M}, \mathrm{Katoh} \mathrm{H}$. Tyrosine kinase activity of EphA2 promotes its $\mathrm{S} 897$ phosphorylation and glioblastoma cell proliferation. Biochem Biophys Res Commun 2018; 499: 920-926, doi: 10.1016/j.bbrc.2018.04.020.

21. Ortiz B, Fabius AW, Wu WH, Pedraza A, Brennan CW, Schultz N, et al. Loss of the tyrosine phosphatase PTPRD leads to aberrant STAT3 activation and promotes gliomagenesis. Proc Natl Acad Sci USA 2014; 111: 8149-8154, doi: 10.1073/pnas.1401952111.

22. Veeriah S, Brennan C, Meng S, Singh B, Fagin JA, Solit DB, et al. The tyrosine phosphatase PTPRD is a tumor suppressor that is frequently inactivated and mutated in glioblastoma and other human cancers. Proc Natl Acad Sci USA 2009; 106: 9435-9440, doi: 10.1073/pnas.090057 1106.

23. Choy CT, Kim H, Lee JY, Williams DM, Palethorpe D, Fellows G, et al. Anosmin-1 contributes to brain tumor malignancy through integrin signal pathways. Endocr Relat Cancer 2013; 21: 85-99, doi: 10.1530/ERC-13-0181.

24. Bae WK, Hong CS, Park MR, Sun EG, Lee JH, Kang K, et al. TAp73 inhibits cell invasion and migration by directly activating KAI1 expression in colorectal carcinoma. Cancer Lett 2018; 415: 106-116, doi: 10.1016/j.canlet.2017.12.002.

25. Kedage V, Selvaraj N, Nicholas TR, Budka JA, Plotnik JP, Jerde $T J$, et al. An interaction with Ewing's sarcoma breakpoint protein EWS defines a specific oncogenic mechanism of ETS factors rearranged in prostate cancer. Cell Rep 2016; 17: 1289-1301, doi: 10.1016/j.celrep.2016. 10.001.

26. Ouyang M, Wang H, Ma J, Lu W, Li J, Yao C, et al. COP1, the negative regulator of ETV1, influences prognosis in triple-negative breast cancer. BMC Cancer 2015; 15: 132, doi: 10.1186/s12885-015-1151-y.

27. Britton E, Rogerson C, Mehta S, Li Y, Li X, Consortium O, et al. Open chromatin profiling identifies AP1 as a transcriptional regulator in oesophageal adenocarcinoma. PLoS Genet 2017; 13: e1006879, doi: 10.1371/journal. pgen.1006879.

28. Gu ML, Wang YM, Zhou XX, Yao HP, Zheng S, Xiang Z, et al. An inhibitor of the acetyltransferases CBP/p300 exerts antineoplastic effects on gastrointestinal stromal tumor cells. Oncol Rep 2016; 36: 2763-2770, doi: 10.3892/or.2016. 5080

29. Tan C, Scotting P. Expression of Kit and Etv1 in restricted brain regions supports a brain-cell progenitor as an origin for cranial germinomas. Cancer Genet 2015; 208: 55-61, doi: 10.1016/j.cancergen.2014.12.007.

30. Keld R, Guo B, Downey P, Cummins R, Gulmann C, Ang $Y S$, et al. PEA3/ETV4-related transcription factors coupled with active ERK signalling are associated with poor prognosis in gastric adenocarcinoma. $\mathrm{Br} J$ Cancer 2011; 105: 124-130, doi: 10.1038/bjc.2011.187.

31. Birner P, Berghoff AS, Dinhof C, Pirker C, Capper D, Schoppmann SF, et al. MAP kinase activity supported by BRAF (V600E) mutation rather than gene amplification is associated with ETV1 expression in melanoma brain 
metastases. Arch Dermatol Res 2014; 306: 873-884, doi: 10.1007/s00403-014-1490-6.

32. Jane-Valbuena J, Widlund HR, Perner S, Johnson LA, Dibner AC, Lin WM, et al. An oncogenic role for ETV1 in melanoma. Cancer Res 2010; 70: 2075-2084, doi: 10.1158/ 0008-5472.CAN-09-3092.

33. Baysan M, Woolard K, Cam MC, Zhang W, Song H, Kotliarova $\mathrm{S}$, et al. Detailed longitudinal sampling of glioma stem cells in situ reveals Chr7 gain and Chr10 loss as repeated events in primary tumor formation and recurrence. Int J Cancer 2017; 141: 2002-2013, doi: 10.1002/ijc.30887.

34. Kosel M, Lachance D, Decker P, Kollmeyer T, Caron A, Drucker K, et al. DLL3 and ETV1 are inactivated/methylated in CIC wild-type, IDH-mutated, 1p/19q-codeleted glioma. Neuro Oncol 2018; 20: vi109, doi: 10.1093/neuonc/noy148. 455.

35. Johnson A, Severson E, Gay L, Vergilio JA, Elvin J, Suh J, et al. Comprehensive genomic profiling of 282 pediatric lowand high-grade gliomas reveals genomic drivers, tumor mutational burden, and hypermutation signatures. Oncologist 2017; 22: 1478-1490, doi: 10.1634/theoncologist.20170242.
36. Cheng T, Wei R, Jiang G, Zhou Y, Lv M, Dai Y, et al. XPNPEP2 is overexpressed in cervical cancer and promotes cervical cancer metastasis. Tumour Biol 2017; 39: 1010428317717122 , doi: $10.1177 / 1010428317717122$.

37. Berx G, van Roy F. Involvement of members of the cadherin superfamily in cancer. Cold Spring Harb Perspect Biol 2009; 1: a003129, doi: 10.1101/cshperspect.a003129.

38. van Roy F. Beyond E-cadherin: roles of other cadherin superfamily members in cancer. Nat Rev Cancer 2014; 14 : 121-134, doi: 10.1038/nrc3647.

39. Terry S, Maille P, Baaddi $H$, Kheuang L, Soyeux P, Nicolaiew $\mathrm{N}$, et al. Cross modulation between the androgen receptor axis and protocadherin-PC in mediating neuroendocrine transdifferentiation and therapeutic resistance of prostate cancer. Neoplasia 2013; 15: 761-772, doi: 10.1593/neo. 122070.

40. Yang X, Chen MW, Terry S, Vacherot F, Chopin DK, Bemis $\mathrm{DL}$, et al. A human- and male-specific protocadherin that acts through the wnt signaling pathway to induce neuroendocrine transdifferentiation of prostate cancer cells. Cancer Res 2005; 65: 5263-5271, doi: 10.1158/0008-5472.CAN05-0162. 